\title{
The treatment and follow up of adult chlamydial ophthalmia
}

\author{
Francesco Carta, Stefania Zanetti, Antonio Pinna, Mario Sotgiu, Giovanni Fadda
}

\begin{abstract}
Sixty two patients diagnosed as having adult chlamydial ophthalmia were treated with oral doxycycline and roxithromycin in association and tetracycline eye wash for 2 weeks. Chlamydial ophthalmia was diagnosed by laboratory detection of the micro-organism in ocular specimens using direct immunofluorescent monoclonal antibody staining for Chlamydia trachomatis, chlamydial culture in cycloheximide treated McCoy cells, and Giemsa staining. An immunoenzymatic method for detection of specific IgG and IgA in patients' serum was used as an additional test to confirm the diagnosis. All patients were reexamined 3 weeks after completing their course of antibiotics and in the case of persistent infection a further course of treatment was given. With this treatment regimen 48 out of 62 patients $(77.4 \%)$ were cured after three courses. Because of the risks of an inadequate response to therapy, we recommend a proper post-treatment follow up in all patients with chlamydial eye infections.
\end{abstract}

(Brf Ophthalmol 1994; 78: 206-208)

Infections by Chlamydia trachomatis are one of the major causes of venereal diseases in industrialised countries. ${ }^{12}$ Adult chlamydial ophthalmia (ACO) usually results from autoinoculation with infected genital secretion ${ }^{2-4}$ and can be clinically indistinguishable from conjunctivitis and keratoconjunctivitis of bacterial or viral aetiology. ${ }^{5}$ Clinical features can include a wide range of symptoms from non-specific conjunctival hyperaemia to corneal changes. ${ }^{67}$ The diagnosis of chlamydial infection is made by the laboratory detection of the micro-organism in ocular specimens. Once diagnosed, ACO should be treated with the appropriate antibiotic therapy. $C$ trachomatis is sensitive to tetracyclines, macrolides (erythromycin, roxithromycin, clarythromycin), rifampicin, sulphonamides, and fluoroquinolones. ${ }^{89}$ The Centers for Disease Control have issued treatment guidelines for $C$ trachomatis infections. ${ }^{10}$ The tetracyclines are the drugs of first choice, whereas macrolides are recommended for pregnant women, young children, and other patients in whom tetracyclines are contraindicated or not tolerated. Because of the frequent occurrence of concomitant genital infection and the relatively slow replication time of the micro-organism, a systemic antibiotic treatment should be used for 2 weeks. $^{510} \mathrm{On}$ its own, topical ocular treatment is usually ineffective; however, it should be given in an attempt to sterilise the ocular secretions more quickly and reduce the risks of transmission of infection.
The aim of this study was to assess the efficacy of a specific treatment for ACO. Owing to the high rate of therapeutic failures we had previously obtained relying on both systemic and topical tetracyclines, we decided to add an erythromycin drug (roxithromycin) to this treatment regimen.

\section{Patients and methods}

Sixty two consecutive patients with ACO, examined at the Institute of Ophthalmology of the University of Sassari, Italy, were included in this study. A standardised questionnaire was completed for each patient to evaluate their clinical history. Ophthalmic examination included slit-lamp biomicroscopy of the anterior segment of the eye and application of fluorescein and rose bengal to the ocular surface to detect any epithelial abnormality. On first examination, all patients underwent the following laboratory investigations for the detection of $C$ trachomatis in ocular specimens.

DIRECT IMMUNOFLUORESCENT MONOCLONAL ANTIBODY STAINING FOR $C$ TRACHOMATIS

The upper palpebral conjunctiva of each eye was scraped with cytology brushes which were then rolled onto slides for direct immunofluorescent monoclonal antibody (DFA) staining (MicroTrak, Syva Co, Palo Alto, CA, USA). Slides were air dried and fixed with acetone. A total of $20 \mu \mathrm{l}$ of fluorescein conjugated monoclonal antibody to $C$ trachomatis was placed over the specimen, which was incubated at $37^{\circ} \mathrm{C}$ for 45 minutes, rinsed several times with phosphate buffered saline ( $\mathrm{pH} 7 \cdot 2$ ), and air dried at room temperature. Slides were read on a Leitz fluorescent microscope (Ernst Leitz, Wetzlar, Germany). A specimen was considered positive for $C$ trachomatis if three or more typical elementary bodies were identified. In the case of chlamydial eye infection, $C$ trachomatis was also looked for in urethral or cervical scrapings with the same technique. The examiner was unware of the results of the other diagnostic tests.

Table 1 Results of chlamydial laboratory tests

\begin{tabular}{lll}
\hline Laboratory test & $\begin{array}{l}\text { Positive } \\
(\text { No }(\%))\end{array}$ & $\begin{array}{l}\text { Negative } \\
(\text { No }(\%))\end{array}$ \\
\hline $\begin{array}{l}\text { Conjunctival scrapings: } \\
\text { Immunofluorescence (DFA) }\end{array}$ & $62(100)$ & - \\
Chlamydial culture & $25(40 \cdot 3)$ & $37(59 \cdot 7)$ \\
$\quad$ Giemsa staining & $30(48 \cdot 3)$ & $32(51 \cdot 7)$ \\
Serological examination: & $12(19 \cdot 3)$ & $50(80 \cdot 7)$ \\
$\quad$ IgG antibodies & $43(69 \cdot 3)$ & $19(30 \cdot 7)$ \\
IgA antibodies & &
\end{tabular}

Correspondence to:

Professor Francesco Carta,

Istituto di Clinica Oculistica,

Università di Sassari, viale $S$

Pietro 43, 07100 Sassari, Italy.

Accepted for publicatio

22 September 1993 


\section{CHLAMYDIAL CULTURE}

Specimens were obtained from the conjunctiva of each eye using Dacron swabs. Each swab was placed in a transport medium containing 4SP. Chlamydial transport vials were immediately frozen to $-70^{\circ} \mathrm{C}$ and thawed after 1 to 4 weeks and cultured. Cultures in cycloheximide treated McCoy cells were processed in the standard fashion. ${ }^{11}$

\section{GIEMSA STAINING}

Conjunctival scrapings for Giemsa staining were processed in the usual manner.

A patient was diagnosed as having ACO if one or more of these laboratory tests were positive.

In addition, blood samples were collected by venepuncture from each patient in order to detect IgG and IgA antibodies to $C$ trachomatis in serum using an immunoenzymatic method (ELISA Chlamydia IgG/IgA, Sclavo Inc, Wayne, NJ, USA). This enzyme linked immunosorbent assay was performed as suggested by the manufacturer. A sample was considered positive for detection of IgG or IgA antibodies if serum absorbance was $20 \%$ higher than the cut off value.

All patients with ACO were treated with oral doxycycline (100 mg daily) and roxithromycin (300 mg daily) in association and tetracycline eye wash (six times daily) for 2 weeks. We recommended patients to inform us promptly about any side effects during the treatment. All of them were re-examined and had conjunctival scrapings for detection of $C$ trachomatis by DFA staining 3 weeks after completing their course of antibiotics. In the case of persistent signs and symptoms of infection and positive DFA staining, a further course of the same treatment was given.

\section{Results}

Sixty two patients ( 27 men, 35 women) with ACO participated in the study. Their ages ranged from 16 to 75 years, with an average of 38.9 years. These patients had conjunctivitis or keratoconjunctivitis of duration ranging from 1 month to 12 years; several had been treated unsuccessfully with non-specific topical antibiotics and steroids for a prolonged period. The mean duration of symptoms was about 1 year when a correct diagnosis was secured. The subjective ocular symptoms were discharge, burning, photophobia, sensation of foreign body, itching, tearing, and blurred vision. Clinically, all patients presented with conjunctival hyperaemia associated with papillary and follicular hyperplasia in the palpebral conjunctiva. Corneal lesions observed included fine epithelial punctate keratitis (eight cases), marginal corneal abscesses similar in appearance to those seen in staphylococcal keratoconjunctivitis (six cases), subepithelial punctate infiltrates clinically similar to those associated with adenovirus infection (three cases), and marginal lower micropannus (two cases).

The results of conjunctival scrapings and serological assay are summarised in Table 1 . All the patients had positive DFA staining. Giemsa
Table 2 Efficacy of therapy on adult chlamydial ophthalmia

\begin{tabular}{clcl}
\hline & $\begin{array}{l}\text { Examined } \\
(\text { No })\end{array}$ & $\begin{array}{l}\text { Recovered } \\
(\text { No }(\%))\end{array}$ & $\begin{array}{l}\text { Not recovered } \\
(\text { No }(\%))\end{array}$ \\
\hline $\begin{array}{c}\text { After 1 course } \\
\text { (partial result) }\end{array}$ & 62 & $22(33 \cdot 5)$ & $40(64 \cdot 5)$ \\
$\begin{array}{c}\text { After 2 courses } \\
\text { (partial result) }\end{array}$ & 40 & $17(42 \cdot 5)$ & $23(57 \cdot 5)$ \\
$\begin{array}{c}\text { After 3 courses } \\
\text { (partial result) }\end{array}$ & 23 & $9(39 \cdot 1)$ & $14(60 \cdot 9)$ \\
$\begin{array}{c}\text { After 3 courses } \\
(\text { total result) }\end{array}$ & 62 & $48(77 \cdot 4)$ & $14(22 \cdot 6)$ \\
\hline
\end{tabular}

stained conjunctival scrapings always showed a mixed inflammatory cell infiltrate; typical basophilic intracytoplasmic inclusion bodies were detected in 30 cases. Positive values of antichlamydial IgG and/or IgA antibodies were found in the sera of 44 out of 62 patients. Microbiological examination in the six patients with marginal corneal abscesses did not show any additional bacterial pathogen in the eyes.

Forty six patients ( 21 men, 25 women) agreed to undergo chlamydial investigation of their genital secretions. Concomitant genital infection was detected in 18 patients (nine men, nine women); the condition was usually asymptomatic.

As shown in Table 2, after three courses of treatment $77 \cdot 4 \%$ (48 out of 62 ) of patients had complete resolution of the disease. Out of the 18 patients with positive conjunctival specimens and negative serology, 17 recovered within two courses of treatment. All 14 patients with marginal corneal abscesses or epithelial punctate keratitis showed complete clinical resolution of their corneal infection after the first course of therapy, although DFA staining was still positive in nine of them. These patients recovered fully after two further courses, and there was no recurrence of infection during the 1 year follow up period. Papillae and subepithelial nonactive infiltrates could be detected for some weeks to some months afterwards, despite complete resolution of all other clinical signs and symptoms and negative DFA staining. None of the patients complained of noteworthy side effects during the treatment.

\section{Discussion}

The clinical differential diagnosis of ACO from conjunctivitis and keratoconjunctivitis of bacterial or viral aetiology is not feasible ${ }^{45712}$; hence, it is necessary to rely on laboratory tests. In this study DFA staining was positive in all patients. The lower rate of positive results with chlamydial culture in comparison with DFA staining was most probably due to the fact that specimens were frozen at $-70^{\circ} \mathrm{C}$ for 1 to 4 weeks until cultured, with loss of viability after thawing. ${ }^{513}$ Giemsa staining was sometimes useful in confirming the diagnosis of chlamydial eye infection. DFA staining is a very simple, quick, and extremely trustworthy technique for detecting chlamydial infections and has been regarded as standard in the laboratory confirmed diagnosis of ACO. ${ }^{451213}$ Therefore, we decided to use this method only in the determination of the patients' response to treatment. We fixed the timing of reexamination at 3 weeks, which is a sufficient 
period for dead Chlamydia to be cleared from conjunctiva in the case of complete eradication of the micro-organism.

Concomitant chlamydial genital infection was detected in 18 out of 46 patients (39.1\%); our results were similar to those found by other authors. ${ }^{214}$ These data agree with the prevalent means of ocular infection: autoinoculation with infected genital secretions. The sexual partners of patients with ACO are very likely to have genital chlamydial infection ${ }^{2}$; hence, to avoid the risks of reinfection, we instructed all patients to refer their partners to appropriate medical facilities for examination and treatment. In the other cases, eye to eye transmission seems the most likely means. ${ }^{2}$ Chlamydial eye infection has also been associated with swimming pool bathing. ${ }^{15}$

It is generally agreed that on its own measurement of chlamydial antibodies is not useful for the diagnosis of chlamydial infection. ${ }^{5}$ Nevertheless, we believe that detection of specific IgG and IgA antibodies in patients' serum may be useful as an additional test to confirm the microbiological diagnosis of chlamydial infection. ${ }^{16}$ Sarov and associated ${ }^{18}$ suggested that in the case of $C$ trachomatis infection the presence of specific serum IgA antibody might be an index of active infection. In our study, negative levels of specific antibodies were found in the serum of 18 out of 62 patients. These patients may be considered as having ACO at a relatively early stage, when pathogen antigens have not yet elicited a strong immune response in serum. ${ }^{16}$

In our area of Italy trachoma was endemic until the 1950s; though, thanks to the improved hygienic and economic conditions, the disease has not been seen for over 30 years. None of the examined patients presented typical clinical findings of trachoma such as pannus located superiorly, limbal follicles, Herbert's peripheral pits, or tarsal conjunctival scarring. ${ }^{19}$ Thus, we believe that in our study we have not dealt with trachoma.

With oral doxycycline and roxithromycin combined with topical tetracycline we achieved about one third of recoveries after each course of antibiotics; on the whole, the success rate after the third course was $77 \cdot 4 \%$. Other authors have reported better treatment success rates after one course of similar therapy. Rapoza and associate $^{20}$ reported complete clinical resolution of all cases of chlamydial conjunctivitis after 2 weeks of treatment with oral tetracycline; in their study, post-treatment chlamydial investigation was not performed. Rönnerstam and associates ${ }^{2}$ reported that all patients with ocular chlamydial infection healed after 9 days of treatment with oral doxycycline $100 \mathrm{mg}$ a day; nine out of 27 patients had post-treatment cultures, which were all negative; chlamydial inclusions were detected in irradiated McCoy cells after iodine staining, which is the least sensitive procedure." The higher rate of therapeutic failure in our study may depend on the fact that we performed post-treatment chlamydial investigation with an extremely trustworthy technique. A high correlation between resolution of the disease and negative conjunctival scrapings was noted, and only patients who, despite the therapy, con- tinued to have ocular signs and symptoms of infection had positive DFA standing. It is worth stressing that 17 of 18 patients with positive conjunctival specimens and negative serology healed after one or two courses of antibiotics. These patients showed few elementary bodies in their conjunctival smears and might be considered as having ACO at an early stage, as suggested by negative serology and their clinical history. In these cases the treatment might have been highly successful because of the relatively short duration of the disease.

In conclusion, because of the risks of an inadequate response to therapy, we recommend that all patients with ACO undergo conjunctival scrapings for detection of $C$ trachomatis by DFA staining at least 3 weeks after completing the course of antibiotics. If a positive DFA result is obtained, patients should be retreated. Antibiotic therapy can often effect clinical cure, but sometimes does not completely eradicate Chlamydia with persistent infection. Long standing infection, reinfection, and a too low antibiotic concentration in conjunctival cells may be a cause of therapeutic failure.

1 Bell TA, Grayston JT. Center for Diseases Control guidelines for prevention of Chlamydia trachomatis infections. Ann Intern Med 1986; 104: 524 .

2 Rönnerstam R, Persson K, Hansson H, Renmarker K. Prevalence of chlamydial eye infection in patients attending an eye clinic, a VD clinic, and in healthy persons. $B r \mathcal{F}$ Ophthalmol 1985; 69 : 385.

3 Insler MS, Anderson AB, Murray M. Latent oculogenital infection with Chlamydia trachomatis. Ophthalmology 1987; 94: 27.

4 Coppens I, Abu El-Asrar AM, Maudgal PC, Missotten L. Incidence and clinical presentation of chlamydial keratoconjunctivitis: a preliminary study. Int Ophthalmol 1988; 12: 201.

5 Taylor HR, Fitch CP, Murillo-Lopez F, Rapoza P. The diagnosis and treatment of chlamydial conjunctivitis. Int diagnosis and treatment

6 Viswalingam ND, Wishart MS, Woodland RM. Adult chlamydial ophthalmia (paratrachoma). Br Med Bull 1983; 39: 123.

7 Darougar S, Viswalingam ND. Marginal corneal abscess associated with adult chlamydial ophthalmia. $\mathrm{Br} \mathcal{F}$ Ophthalmol 1988; 72: 774.

8 Bowie WR. In vitro and in vivo efficacy of antimicrobials against Chlamydia trachomatis. Infect 1982; 10: 46S

$9 \mathrm{Neu} \mathrm{HC}$. Microbiologic aspects of fluoroquinolones. Am $\mathrm{f}$ Ophthalmol 1991; 112: 15S.

10 Centers for Disease Control. Chlamydia trachomatis infection. MMWR 1985; 34: 77S.

11 Stamm WE, Tam M, Koester M, Cles L. Detection of Chlamydia trachomatis in McCoy cell cultures with Chlamydia trachomatis in McCoy cell cultures with Microbiol 1983; 17: 666 .

12 Rapoza PA, Quinn TC, Terry AC, Gottsch JD, Kiessling LA, Taylor HR. A systematic approach to the diagnosis and treatment of chronic conjunctivitis. Am $\mathcal{F}$ Ophthalmol 1990; 109: 138 .

13 Nachamkin I, Sawyer K, Skalina D, Crooks GW, Ciotti R, Sondheimer SJ. Test-of-cure analysis by direct immunfluorescence for Chlamydia trachomatis after antimicrobial therapy. $\mathcal{F}$ Clin Microbiol 1987; 25: 1774.

14 Dawson CR, Schachter J. TRIC agent infections of the eye and genital tract. Am $\mathcal{F}$ Ophthalmol 1967; 63: 1288

15 Huntemüller, Paderstain. Chlamydozoen-Befunde in Schwimmbad-conjunctivitis. Dtsch Med Wochenschr 1913; 36: 63 .

16 Gulletta E, Del Pezzo M, Del Prete A, Covelli I. Laboratory survey of Chlamydia trachomatis ocular infections. Eur $\mathcal{F}$ Epidemiol 1990; 6: 300

17 Cevenini R, Rumpianesi F, Donati M, Sarov I. A rapid immunoperoxidase assay for the detection of specific IgG antibodies to Chlamydia trachomatis. $\mathcal{F}$ Clin Pathol 1983; 36: 353.

18 Piura B, Sarov I, Sarov B, Kleinman D, Chaim W, Insler V. Serum IgG and IgA antibodies specific for Chlamydia trachomatis in salpingitis patients as determined by the immunoperis

19 Thygeson P. Corneal changes in TRIC agent infections. Am $\mathcal{F}$ Ophthalmol 1967; 63: 1278.

20 Rapoza PA, Gottsch JD, Quinn TC, Terry AC, Taylor HR. Etiology and diagnosis of chronic conjunctivitis. Invest Ophthalmol Vis Sci 1986; 27: 34S. 